\title{
Sleep Disturbance in Travelers
}

\section{Reza Bidaki ${ }^{1,2}$, Bonnie Bozorg ${ }^{3 *}$, Kiandokht Khorshidi ${ }^{4}$}

${ }^{1}$ Research Center of Addiction and Behavioral Sciences, Shahid Sadoughi University of Medical Sciences, Yazd, Iran

${ }^{2}$ Diabetes Research Center, Shahid Sadoughi University of Medical Sciences, Yazd, Iran

${ }^{3}$ Tehran University of Medical Sciences, Tehran, Iran

${ }^{4}$ Shahid Sadoughi University of Medical Sciences, Yazd, Iran

Corresponding Author: Bonnie Bozorg, Master of Clinical Psychology, Clinical Psychologist, Tehran University of Medical Sciences, Tehran, Iran. Tel: +98-9121955521. Email: bonnie.bozorg@yahoo.com

Received March 30, 2016; Accepted May 7, 2016; Online Published September 25, 2016

Citation: Bidaki R, Bozorg B, Khorshidi K. Sleep disturbance in travelers. Int J Travel Med Glob Health. 2016;4(3):99. doi:10.20286/ijtmgh-040307.

\section{Dear Editor}

Sleep has biological and circadian rhythms and a complex pattern. When it obeys the biological clock, one's body adapts to the circadian cycle. ${ }^{1}$ The quality and quantity of sleep are important. Insufficient sleep causes impaired attention and alertness, reduced learning, and high stress. Loss of sufficient sleep may cause increased anxiety and hypomania. ${ }^{2}$

Traveling through several time zones causes jet lag. In other words, circadian rhythms adjust slowly and maintain the original biological schedule for few days or even weeks. It is important to remind that among immigrants, acculturation is associated with poor sleep quality in host countries. Several factors influence immigrants, such as background of one's culture (e.g., early life risk factors, diet); factors resulting in the immigration (compulsory or voluntary); factors during the process of immigration; and socioeconomic factors of host country (such as poor socioeconomic conditions or lack of social status). ${ }^{3}$

Sleep disorders such as drowsiness and insomnia have a high instance race in this population group because of changes to their circadian sleep rhythms.

Fatigue, drowsiness, and insomnia are closely related. They cause low alertness, psychological disorders, and forgetfulness, possibly because of a disruption in brain chemicals. People with sleep dissatisfaction can experience low quality of life and a reduction in productivity.

Immigrants to other countries may experience great distress because of sleep disturbance. Jet lag is a direct result of one's ability to traverse several time zones in a short period of time. In addition to night time sleep disturbances and impaired alertness in daytime, general unrest is another symptom of jet lag. ${ }^{4}$ It is even possible that the jet-lagged person will ignore the immigration principals and his/her goals in the face of unpleasant changes. For example, he/she may come to regret his/her action or may develop a negative and biased view toward the new country.

\section{Authors Contributions}

All authors contributed significantly towards the study.

\section{Conflict of Interest Disclosures}

None.

Ethical Approval

Not applicable.

\section{Funding/Support}

There was not funding support for this research.

\section{Acknowledgments}

This topic arises from Dr. Bidakis idea to provoke discussion regarding this issue in subsequent studies.

\section{References}

1. Cooper R. Sleep. London: Chapman \& Hall medical; 1994:94105.

2. Grandner MA, Patel NP, Gehrman PR, Perlis ML, Pack AI. Problems associated with short sleep: bridging the gap between laboratory and epidemiological studies. Sleep Med Rev. 2010;14(4):239-247. doi:10.1016/j.smrv.2009.08.001.

3. Hale L, Troxel WM, Kravitz HM, Hall MH, Matthews KA. Acculturation and sleep among a multiethnic sample of women: the Study of Women's Health Across the Nation (SWAN). Sleep. 2014;37(2):309-17. doi:10.5665/sleep.3404.

4. Diagnostic Classification Steering Committee. International classification of sleep disorders: diagnostic and coding manual. Rochester, MN: American Sleep Disorders Association; 1990:3843. 